\title{
MAXIMAL QUOTIENT RINGS OF PRIME GROUP ALGEBRAS
}

\author{
JOHN HANNAH AND K. C. O'MEARA
}

\begin{abstract}
It is shown that a prime nonsingular group algebra $K G$, where $G$ is a group whose conjugacy classes are countable, has for its maximal right quotient ring either a full linear ring or a simple directly infinite ring. In the case where $G$ is also locally finite only the second type can occur.
\end{abstract}

0. Introduction. The maximal right quotient ring $Q$ of a prime nonsingular ring $R$ is a prime regular right self-injective ring. Accordingly, within the class of such rings, $Q$ falls into one of the following disjoint subclasses (see Goodearl and Handelman [7]):

(a) full linear rings,

(b) nonsimple rings with zero socle,

(c) directly finite non-Artinian rings (necessarily simple),

(d) simple, directly infinite rings.

All four types are possible for a general $R$ (see [7] for examples of (c) and (d); for (b) take $R=T / \operatorname{soc} T$ where $T=\operatorname{Hom}_{F}(V, V)$ for some vector space $V$ over a countable field $F$ with $\operatorname{dim}_{F} V>2^{\kappa_{0}}$ ). However in $\S 1$ we show that if $R$ is a prime nonsingular group algebra $K G$, where $G$ is a group whose conjugacy classes are countable, then only (a) and (d) can occur; type (a) if $K G$ has uniform right ideals, type (d) otherwise. This result is deduced after the corresponding classification is established when $R$ is a countable-dimensional algebra. In $\$ 2$ we show that uniform right ideals do not exist in $K G$ if $G$ is also locally finite, and so the maximal quotient ring in this case is always simple and directly infinite.

The above results are in direct contrast with Kaplansky's result that group algebras over a field of characteristic zero are directly finite. Should some class of group algebras have directly finite maximal quotient rings, this would of course also establish the direct finiteness of these group algebras (and their subgroup-algebras).

Note. Maximal right quotient ring (MRQ ring) is used in the sense of R. E. Johnson [10]. A nonsingular ring is one with zero right singular ideal. Such rings always have an MRQ ring, which is regular and right self-injective. For modules $A$ and $B$ we write $A \leq B$ if $A$ is isomorphic to a submodule of $B$. A ring is directly finite if all one-sided inverses are two-sided (i.e. $x y=1$ implies

Received by the editors December 22, 1975 and, in revised form, April 6, 1976.

AMS (MOS) subject classifications (1970). Primary 16A08, 16A26; Secondary 16A12.

Key words and phrases. Prime nonsingular group algebras, simple maximal right quotient rings, locally finite groups, countable conjugacy classes, directly finite, countable-dimensional algebras. 
$y x=1)$; otherwise it is directly infinite.

1. Countable-dimensional prime algebras. Let $R$ be a prime nonsingular ring. By a well-known result of Johnson [10], if $R$ contains uniform right ideals, then its MRQ ring is the ring of all linear transformations of some vector space (of necessarily countable dimension if $R$ is countable or a countable-dimensional algebra). For $R$ countable but without uniform right ideals we have the following result.

THEOREM 1.1. If $R$ is a countable prime nonsingular ring with no uniform right ideals, then its $M R Q$ ring is simple and directly infinite.

Proof. We shall use the following property of a prime nonsingular ring:

(*) For any nonzero right ideals $A$ and $B$ of $R$, there exist nonzero right ideals $I \subseteq A$ and $J \subseteq B$ with $I \cong J$ (as $R$-modules).

To show the MRQ ring of $R$ is simple and directly infinite, it suffices by Goodearl and Handelman [7, Theorem 5.3] to show that for each nonzero $r \in R$ there exists a large right ideal $L$ of $R$ with $L \leq r R$. We shall establish this by constructing inductively two families $\left\{I_{n}\right\}_{1}^{\infty}$ and $\left\{J_{n}\right\}_{1}^{\infty}$ of independent right ideals of $R$ such that (i) $\Sigma_{1}^{\infty} I_{n}$ is large in $R$, (ii) $\Sigma_{1}^{\infty} J_{n} \subseteq r R$, and (iii) $I_{n} \cong J_{n}$ for all $n$. Taking $L=\Sigma_{1}^{\infty} I_{n}$ gives the desired result.

Construction of $I_{n}, J_{n}$. Since $R$ has no uniform right ideals, there exists a family $\left\{B_{n}\right\}_{1}^{\infty}$ of independent nonzero right ideals of $R$ with $\Sigma_{1}^{\infty} B_{n} \subseteq r R$. Let $a_{1}, a_{2}, \ldots, a_{n}, \ldots$ be an enumeration of the nonzero elements of our countable $R$. Using (*) we choose nonzero right ideals $I_{1}$ and $J_{1}$ of $R$ with

$$
I_{1} \subseteq a_{1} R, \quad J_{1} \subseteq B_{1}, \text { and } I_{1} \cong J_{1} .
$$

Now suppose we have found independent right ideals $I_{1}, \ldots, I_{n}$ and right ideals $J_{1}, \ldots, J_{n}$ with $J_{i} \subseteq B_{i}, I_{i} \cong J_{i}$, and $\left(I_{1}+\cdots+I_{n}\right) \cap a_{i} R \neq 0$ for $i=1, \ldots, n$. If $\left(I_{1}+\cdots+I_{n}\right) \cap a_{n+1} R \neq 0$ we put $I_{n+1}=J_{n+1}=0$. Otherwise we use (*) to find nonzero right ideals $I_{n+1}$ and $J_{n+1}$ with

$$
I_{n+1} \subseteq a_{n+1} R, \quad J_{n+1} \subseteq B_{n+1} \text {, and } I_{n+1} \cong J_{n+1} \text {. }
$$

This establishes the construction.

It is immediate from the construction that (ii) and (iii) hold, and (i) is true because for each $a_{n}$ we have $\left(I_{1}+\cdots+I_{n}\right) \cap a_{n} R \neq 0$. The proof is complete.

By means of the following well-known lemma (see for instance Snider [13, Lemma 3]) we can extend Theorem 1.1 to countable-dimensional prime nonsingular algebras.

Lemma 1.2. Suppose $R$ is an algebra over a field $F$ and that $K$ is a field extension of $F$. If $L$ is a large right ideal of $R$ then $K \otimes_{F} L$ is a large right ideal of $K \otimes_{F} R$. (So if $K \otimes_{F} R$ is nonsingular then so is $R$.)

THEOREM 1.3. Let $R$ be a prime nonsingular countable-dimensional algebra 
over a field $K$. If $R$ has no uniform right ideals, then its $M R Q$ ring is simple and directly infinite.

Proof. By [7, Theorem 5.3] the MRQ ring is simple and directly infinite if for each $r \in R, r \neq 0$, there exists a large right ideal $L$ of $R$ with $L \leqslant r R$. Let $r$ be given. Since $R$ has no uniform right ideals, there exists a family $\left\{b_{n} R\right\}_{1}^{\infty}$ of independent nonzero principal right ideals of $R$ such that $\Sigma_{1}^{\infty} b_{n} R \subseteq r R$. Let $\left\{x_{i}\right\}_{1}^{\infty}$ be a $K$-basis for $R$. Then there exists a countable subfield $F$ of $K$ and an $F$-subalgebra $S$ of $R$ such that $\left\{x_{i}\right\}_{1}^{\infty}$ is an $F$-basis for $S, r \in S$, and $b_{n} \in S$ for all $n$. Since $R=K S$ is prime, clearly $S$ is prime. By $1.2, S$ is nonsingular. Thus $S$ is a countable prime nonsingular ring and we have $\bigoplus \Sigma_{1}^{\infty} b_{n} S \subseteq r S$. Hence by the proof of 1.1 (taking $B_{n}=b_{n} S$ ), there exists a large right ideal $L_{1}$ of $S$ and an $S$-monomorphism $\psi: L_{1} \rightarrow r S$. Let $L=K L_{1}$. By $1.2, L$ is a large right ideal of $R$. Since $\psi$ extends to an $R$-monomorphism $\bar{\psi}: K L_{1} \rightarrow r R$ we have $L \leqslant r R$, as desired.

As an immediate corollary to 1.3 we have:

COROLlaRY 1.4. Let $G$ be a countable group with $\Delta^{+}(G)=1$, and let $K$ be any field for which $K G$ is nonsingular. If $K G$ contains no uniform right ideals, then its $M R Q$ ring is simple and directly infinite.

Note. $\Delta^{+}(G)$ is the torsion subgroup of the f.c. subgroup of $G$. A wellknown result of $\mathrm{I}$. G. Connell characterizes prime group algebras $K G$ as those for which $\Delta^{+}(G)=1$. In the sequel, we call a group $G$ prime if $\Delta^{+}(G)=1$. For conditions under which $K G$ is nonsingular, see Example 2.6.

1.4 can be extended to prime groups whose conjugacy classes are countable. To establish this we need two lemmas.

LEMMA 1.5. Let $G$ be a prime group whose conjugacy classes are countable. Then for any countable subgroup $H$ of $G$, there exists a countable prime normal subgroup $\bar{H}$ of $G$ containing $H$.

Proof. If $A \triangleleft G$ and $B$ is a subgroup containing $A$, let us say that the conjugacy classes of $A$ are 'fused' in $B$ if each $B$-conjugacy class contained in $A$ is a complete $G$-conjugacy class. We shall construct inductively a countable family $\left\{H_{n}\right\}_{1}^{\infty}$ of countable normal subgroups of $G$ containing $H$ such that $H_{n} \subseteq H_{n+1}$ and the classes of $H_{n}$ are fused in $H_{n+1}$ for all $n$. Taking $\vec{H}=\cup_{1}^{\infty} H_{n}$ then gives the desired result. ( $\bar{H}$ is prime because $G$ is prime and the classes of $\bar{H}$ are already fused in $\bar{H}$.)

To begin the construction, we let $H_{1}$ be the normal closure of $H$. Since the conjugacy classes of $G$ are countable, the normal closure (in $G$ ) of any countable subgroup of $G$ is again clearly countable. Now suppose we have constructed $H_{1}, \ldots, H_{n}$. Since $H_{n}$ is countable there exists a countable subset $C_{n}$ of $G$ such that the classes of $H_{n}$ are fused in $\left\langle H_{n}, C_{n}\right\rangle$. Now for $H_{n+1}$ we take the normal closure of $\left\langle H_{n}, C_{n}\right\rangle$. This establishes the construction. 
LEMMA 1.6. If $H$ is a normal (or subnormal) subgroup of $G$, then any large right ideal of $K H$ generates a large right ideal of $K G$. (In particular if $K G$ is nonsingular then so is $\mathrm{KH}$.)

Proof. See Burgess $[1,2.5]$.

THEOREM 1.7. Let $G$ be a prime group whose conjugacy classes are countable, and let $K$ be any field for which $K G$ is nonsingular. If $K G$ has no uniform right ideals, then its $M R Q$ ring is simple and directly infinite.

Proof. Let $R=K G$ and let $0 \neq r \in R$. It suffices to produce a large right ideal $L$ of $R$ with $L \leq r R$. Let $\left\{b_{n} R\right\}_{1}^{\infty}$ be as in the proof of 1.3. Applying 1.5 to the subgroup generated by the supports of the $b_{n}$ and $r$, we can find a countable prime normal subgroup $H$ of $G$ such that $r, b_{n} \in K H$ for all $n$. Now $K H$ is a countable-dimensional prime nonsingular algebra, and we have $\bigoplus \Sigma_{1}^{\infty} b_{n} K H \subseteq r K H$. Hence by the proof of 1.3 , there exists a large right ideal $I$ of $K H$ and a $K H$-monomorphism $\psi: I \rightarrow r K H$. Let $L=I R$. By $1.6, L$ is a large right ideal of $R$. Since $\psi$ extends to a $K G$-monomorphism $\bar{\psi}: I R \rightarrow r R$, we have $L \leq r R$ as desired.

REMARK. The proof of 1.7 used the existence of a suitable countable $H$ such that large right ideals of $K H$ generate large right ideals of $K G$. This property is not shared by all subgroups, as shown by the following example due to Formanek [4]: let $G$ be the free group on two generators $a$ and $b$. Let $H=\langle a\rangle$. Then $(1-a) K H$ is large in $K H$ but $(1-a) K G$ is not large in $K G$ because $(1-a) K G \cap(1-b) K G=0$.

2. Locally finite group algebras. For groups whose conjugacy classes are countable, our attempt to classify the MRQ rings of prime group algebras requires only a criterion for the existence of uniform right ideals. In this section we shall supply that need in the case of locally finite groups by using the following (probably well-known) dimension function.

Let $K$ be a field and $G$ a locally finite group, and denote by $L(K G)$ the lattice of finitely generated right ideals of $K G$. Given $A=a_{1} K G+\cdots+$ $a_{n} K G \in L(K G)$ we find $d(A)$ as follows: let $H$ be a finite subgroup of $G$ containing the supports of $a_{1}, \ldots, a_{n}$ and write

$$
d(A)=|H|^{-1} \operatorname{dim}_{K}\left(a_{1} K H+\cdots+a_{n} K H\right) .
$$

Proposition 2.1. If $G$ is locally finite then $d: L(K G) \rightarrow[0,1]$ is well defined, and if $A, B \in L(K G)$ then

(i) $d(A) \geqslant 0$ with equality if and only if $A=0$;

(ii) $d(A) \leqslant 1$ with equality if and only if $A=K G$,

(iii) $A \leq B$ implies $d(A) \leqslant d(B)$,

(iv) $A \cap B=0$ implies $d(A+B)=d(A)+d(B)$.

Proof. That $d(A)$ is independent of the choice of $H$ follows because if $g_{1}, \ldots, g_{m}$ is a complete set of right coset representatives for $\dot{H}$ in some 
finite subgroup $H_{1}$ then

$$
\sum_{i=1}^{n} a_{i} K H_{1}=\sum_{j=1}^{m}\left(\sum_{i=1}^{n} a_{i} K H\right) g_{j}
$$

and the sum over $j$ is a direct sum of isomorphic $K$-modules. It is now easy to see that $d(A)$ is independent of the choice of $a_{1}, \ldots, a_{n}$ and that $d$ satisfies (i) to (iv).

We shall use $d$ to show that locally finite prime group algebras have zero socle. Müller (see [11]) has already shown that many locally finite group algebras have zero socle; for example, those $K G$ where $G$ is infinite and $K$ is algebraically closed, or where $G$ has the property that, for any finite subgroup $U$, there is a finite nontrivial subgroup $V$ normalized by $U$ and satisfying $U \cap V=1$. We do not know if this latter class includes all prime groups and so, since Müller also shows that some locally finite group algebras have nonzero socle, we avoid that approach as follows.

THEOREM 2.2. If $G \neq 1$ is a locally finite prime group then soc $K G=0$.

Proof. Since $G$ is prime it is infinite. By considering right ideals of the form $\left(h_{1}+\cdots+h_{n}\right) K G$, where $\left\{h_{1}, \ldots, h_{n}\right\}$ is a subgroup of $G$, we see that $\inf \{d(A): A \neq 0\}=0$. Suppose however that $A \neq 0$ is a minimal right ideal and let $0 \neq B \in L(K G)$. As $K G$ is prime, $A \leq B$ and so $d(A) \leqslant d(B)$. This gives the contradiction $0<d(A)=\inf \{d(B): B \neq 0\}$.

We can now prove the main result of this section.

THEOREM 2.3. Let $G \neq 1$ be a locally finite prime group whose conjugacy classes are countable. If $K G$ is nonsingular then its $M R Q$ ring is simple and directly infinite.

Proof. Since $G$ is locally finite, the radical and the singular ideal of $K G$ coincide by Proposition 9 of [13]. Hence $K G$ is semisimple and so, as in Lemma 2.1 of [2], every nonzero right ideal of $K G$ contains a nonzero idempotent. Thus any uniform right ideal of $K G$ is minimal. Our theorem now follows from Theorems 2.2 and 1.7.

Theorem 2.3 is an interesting parallel to Fisher and Snider's result (see [2]) that such group algebras are primitive if $G$ is countable. At the same time, it contrasts with Goursaud and Valette's result (see [9]) that, for suitable $K$, if $K G$ is regular then the type I part of the MRQ ring of $K G$ is directly finite.

We do not know if Theorem 2.3 remains true when the countability hypothesis is dropped. However, the first part of our next result shows that, because of $d$, right ideals of $K G$ cannot be "too big" when $G$ is locally finite. We shall use this to prove that in general the MRQ ring of $K G$ is at most one step away from being simple. The second part of the result generalizes Proposition 2.11 of [8].

Proposition 2.4. Let $G$ be locally finite. Then every right ideal of $K G$ is an 
essential extension of some countably generated right ideal. Consequently $K G$ is hereditary if and only if $K G$ is regular and $G$ is countable.

Proof. The first statement is proved by using $d$ and imitating Lemma 13 of [6]. Suppose $K G$ is hereditary. By Lemma 2.1 and Proposition 2.2 of [8], $K G$ is regular. Now $\omega G$ is a projective right ideal which is an essential extension of a countably generated right ideal. As $K G$ is nonsingular, $\omega G$ is itself countably generated and so $G$ is countable. The converse is clear.

REMARK. The second statement also follows from the proof of Proposition 2.11 of [8] because if $H$ is any infinite subgroup of $G$ then $\sup \{d(A)$ : $A \subseteq \omega H\}=1$ and this is enough to make $\omega H$ large.

Proposition 2.5. If $G$ is a locally finite prime group and $K G$ is nonsingular, then the $M R Q$ ring $Q$ of $K G$ has at most one proper ideal. If, in addition, $G$ has a prime subnormal subgroup $H$ whose conjugacy classes are countable then $Q$ is directly infinite.

Proof. By the first part of Proposition 2.4, $K G$ has no uncountable families of independent nonzero right ideals. Hence, in the terminology of [5], $H\left(\kappa_{1}\right)$ $=Q$ and $Q$ has at most one proper ideal, namely $H\left(\kappa_{0}\right)$. The second part follows because, by [4], the MRQ ring of $K H$ is contained in $Q$ and because of Theorem 2.3.

Finally we mention just a few of the many known interesting examples of prime nonsingular group algebras.

EXAMPLE 2.6. (1) char $K=0$. By Theorem 4 of [13], $K G$ is nonsingular for any group $G$. So if $\Delta^{+}(G)=1$ then $K G$ is a prime nonsingular group algebra. In particular $G$ can be chosen to be one of the following groups:

(a) the group $S_{X}$ of all permutations of an infinite set $X$ which move only finitely many elements of $X$ (if $X$ is countable then, by Theorem 2.3, the MRQ ring of $K G$ is simple and directly infinite),

(b) the (weak) wreath product $A \sim B$ where $A$ is nontrivial and $B$ is infinite (see [12, Lemma 21.5]),

(c) any polycyclic (or for that matter, abelian-by-finite) group with $\Delta^{+}(G)$ $=1$ (for example, the infinite dihedral group); in this case Goldie's theorem (or Posner's, respectively) shows that the MRQ ring of $K G$ is a finite-dimensional full linear ring since $K G$ is prime and Noetherian (or prime and satisfies a polynomial identity, respectively) (see [12, Theorem 5.1 and p. 136]),

(d) any infinite simple group.

(2) $\operatorname{char} K^{\prime}=p>0$.

(i) If $G$ is locally finite the radical and singular ideal of $K G$ coincide and so prime semisimple $K G$ provide further examples. In particular, by [3], we could use the permutation group $S_{X}$ (see (a) above).

(ii) If $G$ is solvable then $K G$ is nonsingular if and only if $G$ has no finite subnormal subgroups of order divisible by $p$ (see [13, Theorem 7]). 
ACKNOWLEDGEMENT. The authors wish to thank the referee for his helpful suggestions.

NOTE ADDED IN PROOF. In a forthcoming paper, The socle in group rings, B. Hartley and J. S. Richardson characterize the locally finite group algebras with nonzero socle (cf. Theorem 2.2).

\section{REFERENCES}

1. W. D. Burgess, Rings of quotients of group rings, Canad. J. Math. 21 (1969), 865-875. MR 39 \# 5714.

2. J. W. Fisher and R. L. Snider, Prime von Neumann regular rings and primitive group algebras, Proc. Amer. Math. Soc. 44 (1974), 244-250. MR 49 \# 7297.

3. E. Formanek, A problem of Passman on semisimplicity, Bull. London Math. Soc. 4 (1972), 375-376. MR 47 \#3439.

4. __ Maximal quotient rings of group rings, Pacific J. Math. 53 (1974), 109-116.

5. K. R. Goodearl, Prime ideals in regular self-injective rings, Canad. J. Math. 25 (1973), 829-839. MR 48 \# 4037.

6. Simple regular rings and rank functions, Math. Ann. 214 (1975), 267-287.

7. K. R. Goodearl and D. Handelman, Simple self-injective rings, Comm. Algebra 3 (1975), 797-834.

8. J. M. Goursaud and J. Valette, Anneaux de groupe héréditaires et semi-héréditaires, J. Algebra 34 (1975), 205-212.

9. , Sur l'enveloppe injective des anneaux de groupes reguliers, Bull. Soc. Math. France 103 (1975), 91-102.

10. R. E. Johnson, Quotient rings of rings with zero singular ideal, Pacific J. Math. 11 (1961), 1385-1392. MR 26 \#1331.

11. W. Müller, Radikal und Sockel in Gruppenalgebren über lokalendlichen Gruppen, Arch. Math. (Basel) 25 (1974), 476-482. MR 50 \# 10034.

12. D. S. Passman, Infinite group rings, Pure and Appl. Math., no. 6, Dekker, New York, 1971. MR 47 \#3500.

13. R. L. Snider, On the singular ideal of a group algebra, Comm. Algebra 4 (1976), 1087-1089.

Department of Mathematics, University of Canterbury, Christchurch 1, New Zealand 